\title{
TESTE DE REPELÊNCIA DE ÓLEOS ESSENCIAIS SOBRE CALLOSOBRUCHUS MACULATUS
}

\author{
Antonia Mirian Nogueira de Moura Guerra1*, Deyse dos Santos Silva2, Priscila Silva Santos3, Lucas Barbosa dos \\ Santos4
}

\begin{abstract}
RESUMO - Este trabalho foi realizado na Universidade Federal do Oeste da Bahia campus Barra, com o objetivo de avaliar o efeito repelente dos óleos essenciais de plantas medicinais, como método de controle do bruchidio Callosobruchus maculatus. O efeito repelente dos óleos essenciais de Capim-santo (Cymbopogon citratus) e Erva cidreira brasileira (Lippia alba) e do extrato etanólico de Malvão (Plectranthus amboinicus) foi testado sobre os adultos de C. maculatus. Os óleos essenciais foram obtidos em extração por arraste de vapor utilizando o aparelho de extração tipo Clevenger. Já o extrato etanólico Malvão foi obtido a partir da maceração de $100 \mathrm{~g}$ de folhas e imersão em $200 \mathrm{~mL}$ de etanol $70 \%$, e após $48 \mathrm{~h}$ foi realizada a filtragem. Utilizaram-se placas de Petri de $90 \mathrm{~mm}$ de diâmetro contendo discos de papel filtro divididos ao meio para a aplicação dos óleos essenciais/extrato etanólico, sendo aplicados em uma das metades e na outra aplicouse apenas água destilada como controle. Após a secagem foram liberados 10 insetos adultos não sexados, registrando, após 24 h a sua distribuição. Para o estudo de repelência foram utilizadas as doses de 20, 40, 60, 80 e $100 \mu \mathrm{L}$. Nos ensaios de mortalidade, utilizou-se um tempo fixo de $24 \mathrm{~h}$ para a avaliação, e avaliou-se a variação nas doses dos óleos/extrato sobre a mortalidade dos insetos. Seguiu-se a mesma metodologia de aplicação dos óleos essenciais/extrato sobre discos de discos de papel filtro nas placas de Petri. Adotaram-se as doses de $0,20,40,60,80$ e $100 \mu \mathrm{L}$ do óleo/extrato etanólico. Os testes foram realizados em uma temperatura ambiente de $27 \pm 2{ }^{\circ} \mathrm{C}$ e uma umidade relativa do ar de $70 \pm 5 \%$. Foram estimadas as doses letais para 30, 50, 70 e $90 \%$ dos insetos adultos para cada espécie. Seguiu-se um esquema fatorial com seis repetições num delineamento inteiramente casualizado. As espécies avaliadas neste estudo apresentaram efeito repelente e potencial inseticida, causando mortalidades acima de 50\% e Índices de Repelência (IR) de aproximadamente $100 \%$ sobre o C. maculatus. O óleo essencial de $L$. alba o foi mais eficiente nas doses superiores a $40 \mu \mathrm{L}$ ocasionando maior IR. Os óleos essenciais de C. citratus e $L$. alba ocasionaram mortalidades superiores a $70 \%$. Observou-se que os óleos essenciais apresentaram toxidade aguda tópica para os gorgulhos $C$. maculatus: C. citratus $\left(\mathrm{DL}_{50}=13,67 \mu \mathrm{L}\right)$, L. alba $\left(\mathrm{DL}_{50}=13,28 \mu \mathrm{L}\right)$ e P. amboinicus $\left(\mathrm{DL}_{50}=12,89 \mu \mathrm{L}\right)$. Os óleos essenciais e o extrato etanólico das plantas estudadas apresentaram toxicidade ao gorgulho-do-feijão, podendo, serem utilizados no controle desta praga durante o armazenamento.
\end{abstract}

Palavras chave: Cymbopogon citratus, Lippia alba, Plectranthus amboinicus.

\footnotetext{
${ }^{1}$ Docente do curso de Agronomia da Universidade Federal do Oeste da Bahia, Centro Multidisciplinar Campus de Barra, Av. 23 de Agosto s/nº, Bairro Assunção, CEP: 47100-000, Barra - BA. E-mail: mirianagronoma@hotmail.com. *Autor para correspondência.

${ }^{2}$ Discente do curso de Agronomia da Universidade Federal do Oeste da Bahia, Centro Multidisciplinar Campus de Barra, Av. 23 de Agosto s/nº, Bairro Assunção, CEP: 47100-000, Barra - BA.

${ }^{3}$ Discente do curso de Agronomia da Universidade Federal do Oeste da Bahia, Centro Multidisciplinar Campus de Barra, Av. 23 de Agosto s/nº, Bairro Assunção, CEP: 47100-000, Barra - BA.

${ }^{4}$ Discente do curso de Agronomia da Universidade Federal do Oeste da Bahia, Centro Multidisciplinar Campus de Barra, Av. 23 de Agosto s/n ${ }^{\circ}$, Bairro Assunção, CEP: 47100-000, Barra - BA.
} 


\title{
ESSENTIAL OIL REJECTION TEST ON CALLOSOBRUCHUS MACULATUS
}

\begin{abstract}
The aim of this study was to evaluate the repellent effect of essential oils of medicinal plants as a method of control of Bruchidium Callosobruchus maculatus. The repellent effect of the essential oils of saint grass (Cymbopogon citratus) and Brazilian lemon grass (Lippia alba) and of the ethanolic extract of Malva (Plectranthus amboinicus) was tested on adults of C. maculatus. The essential oils were obtained by steam extraction using the Clevenger extraction apparatus. The Malanol ethanol extract was obtained from the maceration of $100 \mathrm{~g}$ of leaves and immersion in $200 \mathrm{~mL}$ of $70 \%$ ethanol, and after $48 \mathrm{~h}$ the filtration was performed. $90 \mathrm{~mm}$ diameter Petri dishes containing filter paper disks divided in halffor the application of the essential oils/ethanolic extract were applied in one half and in the other only distilled water was applied as control. After drying, ten adult non-sexed insects were released, registering their distribution after 24 hours. For the study of repellency, the doses of 20,40,60,80 and $100 \mu \mathrm{L}$ were used. In the mortality trials, a fixed time of $24 \mathrm{~h}$ was used for the evaluation, and the variation in the doses of the oils/extract on the mortality of the insects was evaluated. The same methodology of application of the essential oils/extract on discs of filter paper disks in Petri plates was followed. The doses of 0, 20, 40, 60, 80 and $100 \mu \mathrm{L}$ of the oil/ethanolic extract were adopted. The tests were performed at an ambient temperature of $27 \pm 2{ }^{\circ} \mathrm{C}$ and an air relative humidity of $70 \pm 5 \%$. Lethal doses were estimated for 30, 50, 70 and $90 \%$ of adult insects for each species. A factorial scheme with six replicates was followed in a completely randomized design. The species evaluated in this study had a repellent effect and insecticidal potential, causing mortalities above 50\% and Repellency Indices (IR) of approximately 100\% on C. maculatus. The essential oil of L. alba was more efficient at doses higher than $40 \mu L$, resulting in higher IR. The essential oils of C. citratus and L. alba caused mortality higher than 70\%. It was observed that the essential oils presented acute toxicity topical for $C$. maculatus weeds: $C$. citratus $(L D 50=13.67 \mu L)$, L. alba $(L D 50=13.28 \mu L)$ and P. amboinicus $(L D 50=12.89 \mu L)$. The essential oils and the ethanolic extract of the studied plants presented toxicity to the bean weevil, being able to be used in the control of this pest during the storage.
\end{abstract}

Keywords: Cymbopogon citratus, Lippia alba, Plectranthus amboinicus.

\section{INTRODUÇÃO}

O armazenamento de grãos e outros produtos agrícolas visa manter as características qualitativas e quantitativas dos mesmos durante períodos prolongados de tempo (Quirino, 2011; Melo et al., 2011).

Existem inúmeros fatores que podem interferir no estado dos produtos durante o período de armazenamento, ocasionando perdas das características dos mesmos. Dentre eles, encontram-se os insetos-praga de grãos armazenados (Scheepens et al., 2011).

Entre as principais espécies de insetos que atacam o feijão na fase pós-colheita o Callosobruchus maculatus destaca-se como a principal praga de grãos de feijão caupi (Vigna unguiculata (L.) Walp.), durante o armazenamento. Estes insetos atacam o feijão reduzindo o peso, a qualidade dos grãos e seu valor nutritivo. Além disso, a presença de ovos ou de insetos adultos acarreta na desvalorização comercial do produto (Lima et al., 2001; Silva, 2011).
É sabido que a utilização de produtos químicos vem contribuindo há várias décadas para o aumento da produção agrícola, bem como para o controle de pragas de armazenamento. No entanto, o uso incorreto e indiscriminado destes produtos trouxe também muitos males (Corrêa \& Salgado, 2011). Os problemas decorrentes da utilização indiscriminada de produtos químicos apontam para a necessidade de se desenvolver novos tipos de agentes de controle mais seletivos e menos agressivos ao homem e ao ambiente (Kim et al., 2003; Menezes, 2005; Corrêa \& Salgado, 2011). Assim, os produtos botânicos surgem como uma alternativa eficiente para o controle de pragas, uma vez que há uma demanda crescente por alimentos saudáveis e isentos de resíduos de produtos químicos.

Os óleos essenciais são descritos como misturas de substâncias voláteis, geralmente odoríficas e líquidas obtidas a partir de folhas, raízes, cascas e sementes. Suas principais características são o cheiro e o sabor (Barroso, 2009; Fernandes \& Favero, 2014). Também são denominados óleos voláteis por possuírem hidrocarbonetos 
terpênicos, aldeídos, cetonas, fenóis, ésteres, álcoois simples, entre outros. Muitas dessas moléculas apresentam comportamento tóxico ou de repelência interferindo no sistema nervoso do inseto (Estrela et al., 2006; Fernandes \& Favero, 2014).

Recentes investigações em vários países confirmam que alguns óleos essenciais de plantas têm a capacidade de repelir insetos, e apresentam também ação inseticida através do contato direto ou pelas vias respiratórias dos insetos (Corrêa \& Salgado, 2011).

As espécies Cymbopogon citratus, Lippia alba e Plectranthus amboinicus, assim como muitas outras espécies vegetais, são utilizadas empiricamente por apresentarem propriedades terapêuticas. Muitas dessas propriedades são atribuídas a presença de óleos essenciais que podem também ser usados para diversas finalidades (Bandeira et al., 2011). Sendo assim, o objetivo desse trabalho foi avaliar o efeito repelente dos óleos essenciais e extrato etanólico de plantas medicinais, como método de controle do Callosobruchus maculatus (F.).

\section{MATERIAL E MÉTODOS}

O efeito repelente do óleo essencial das espécies vegetais Capim-santo (Cymbopogon citratus) e Erva cidreira brasileira (Lippia alba) e o extrato etanólico de Malvão (Plectranthus amboinicus) foi testado em bioensaios com adultos de C. maculatus.

Os óleos essenciais foram obtidos a partir das folhas das plantas. Para as espécies Capim-santo (Cymbopogon citratus) e Erva cidreira brasileira (Lippia alba), foram trituradas em liquidificador $300 \mathrm{~g}$ de folhas em $1 \mathrm{~L}$ de água destilada, por aproximadamente 3 minutos, sendo esse sistema o mais eficaz para a extração de óleo essencial, segundo Conte et al. (2001). Para a obtenção do óleo essencial foi utilizada a técnica de hidrodestilação com aparelho de extração tipo Clevenger. O material vegetal triturado no liquidificador ficou em contato direto com a água fervente por duas horas. O óleo essencial foi volatizado juntamente com vapores de água e condensado em um sistema fechado, onde posteriormente, a camada do óleo foi separada da fase aquosa (Saito \& Scramin, 2000).

Para a espécie Malvão (Plectranthus amboinicus), foi utilizado o extrato etanólico de em virtude da dificuldade de extração do óleo pelo método do Clevenger, única ferramenta disponível para a obtenção dos óleos essenciais.
O extrato etanólico foi obtido a partir de $100 \mathrm{~g}$ de folhas maceradas e imersas em $200 \mathrm{~mL}$ de etanol 70\%. Após 48 $\mathrm{h}$ foi realizada a filtragem, descartando-se os materiais sólidos.

Os insetos foram obtidos de criações mantidas no Laboratório da UFOB - Barra, os quais foram criados em frascos de vidro de 1,0 L sob condições constantes de temperatura $\left(30^{\circ} \mathrm{C}\right)$ e umidade relativa $(70 \%)$ e escotofase de 24 horas. Como substrato alimentar, foram utilizados grãos de feijão caupi inteiros com teor de água de 13\% b.u., previamente expurgados com fosfina e mantidos sob refrigeração $\left(-18^{\circ} \mathrm{C}\right)$ para evitar reinfestação. Foram realizados os bioensaios de Repelência, utilizando-se, para isso, adultos de Callosobruchus maculatus com 2-7 dias de idade, não sexados, conforme o método descrito por Conte et al. (2002).

\section{Teste de repelência}

Utilizaram-se placas de Petri com discos de papel filtro número 2 com 90 mm de diâmetro, divididos ao meio para a aplicação dos óleos essenciais/extrato etanólico que foram testados. Em uma das metades aplicou-se a dose de óleo essencial/extrato e na outra aplicou-se apenas água destilada como controle. Após a secagem, os discos foram colocados em placas de Petri com mesmo diâmetro (Conte \& Favero, 2001). Em seguida, foram liberados 10 insetos adultos não sexados no centro de cada placa, registrando, após 24 h a distribuição desses insetos. Os testes foram realizados em uma temperatura ambiente de $27 \pm 2{ }^{\circ} \mathrm{C}$ e uma umidade relativa do ar de $70 \pm 5 \%$.

Foram utilizadas as doses de 20, 40, 60, 80 e $100 \mu \mathrm{L}$ do óleo essencial e do extrato etanólico em cada unidade experimental. Seguiu-se um esquema fatorial 3x5 (três espécies vegetais e cinco doses) com seis repetições, num delineamento inteiramente casualizado. equação:

O índice de repelência (IR\%) foi calculado pela

$$
\operatorname{IR}(\%)=100-\left[\left(\frac{T}{T+C}\right) \cdot 100\right]
$$

Onde:

$$
\begin{aligned}
& \text { IR (\%) = índice de repelência. } \\
& \text { T = número de insetos sobre a superfície tratada. } \\
& \text { C = número de insetos sobre a superfície controle. }
\end{aligned}
$$




\section{Teste de mortalidade}

Os óleos e o extrato etanólico também foram avaliadas em ensaios de dose resposta para mortalidade dos insetos adultos. Utilizou-se um tempo fixo de $24 \mathrm{~h}$ para a avaliação, estabelecida com base no estudo de repelência, em que foi constatada mortalidade total da população, e investigou-se a variação nas doses dos óleos/extrato sobre a mortalidade dos insetos.

Seguiu-se a mesma metodologia de aplicação dos óleos essenciais/extrato etanólico sobre discos de discos de papel filtro sobre as placas de Petri, desta vez não dividindo em duas partes, e sim dispondo o óleo/extrato ao centro do papel. Adotaram-se as doses de 0, 20, 40, 60, 80 e $100 \mu \mathrm{L}$ do óleo/extrato etanólico. Em seguida, foram liberados 10 insetos adultos não sexados no centro de cada placa, registrando, após 24 h a mortalidade destes. Foram estimadas as doses letais para 30, 50, 70 e 90\% dos insetos adultos em cada espécie estudada. Seguiu-se um esquema fatorial 3x6 (três espécies vegetais e seis doses) com seis repetições, num delineamento inteiramente casualizado. Os testes foram realizados em uma temperatura ambiente de $27 \pm 2{ }^{\circ} \mathrm{C}$ e uma umidade relativa do ar de $70 \pm 5 \%$.

Os dados foram submetidos à análise de variância e ajustados modelos matemáticos para explicar o comportamento de cada espécie.

\section{RESULTADOS E DISCUSSÃO}

Houve interação entre as espécies vegetais e as doses utilizadas apresentando efeito significativo sobre o Índice de Repelência (IR) dos insetos (Figura 1). Para a espécie $C$. citratus foi constatado maior IR quando aplicado nas doses de 40 a $80 \mu \mathrm{L}$. Já a $L$. alba foi mais eficiente a partir da dose de $40 \mu \mathrm{L}$. Para o extrato etanólico da espécie Plectranthus amboinicus (Malvão) o efeito repelente foi significativo a partir da dose de $20 \mu \mathrm{L}$, reduções nas doses de 60 e $80 \mu \mathrm{L}$, com maior mortalidade da dose de $100 \mu \mathrm{L}$ (Figura 1).

No que se refere a mortalidade de $C$. maculatus submetidos a exposição aos óleos essenciais e extrato etanólico, houve efeito significativo a interação entre as espécies e as doses utilizadas (Figura 2). Entre as espécies isoladamente, Cymbopogon citratus e Lippia alba apresentaram mortalidades de $70,83 \%$ e $71,38 \%$, respectivamente, não havendo diferença significativa entre elas. A espécie Plectranthus amboinicus apresentou menor mortalidade (53\%) de insetos quando comparada com as demais espécies (Tabela 1).

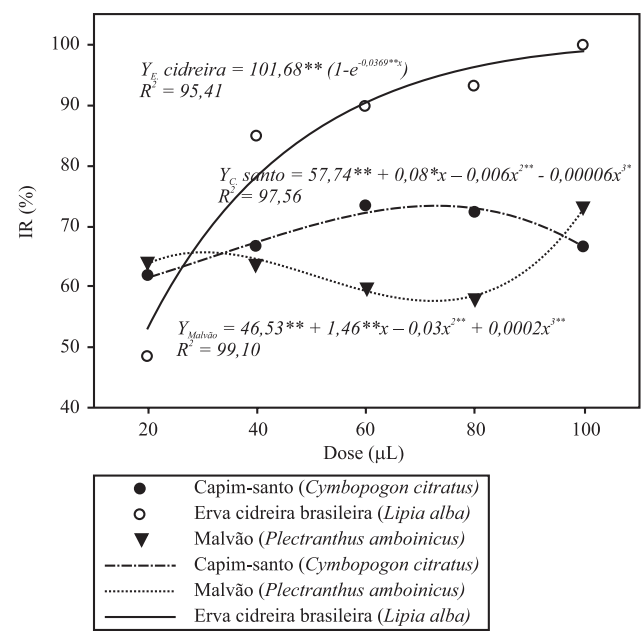

Figura 1 - Índice de Repelência (IR) de adultos de Callosobruchus maculatus (F.) submetidos a exposição aos óleos essenciais de Capimsanto (Cymbopogon citratus), Erva cidreira brasileira (Lippia alba) e extrato etanólico de Malvão (Plectranthus amboinicus) nas doses de $20,40,60,80$ e $100 \mu \mathrm{L}$ ao longo de 24 h de exposição. Os símbolos representam as médias de seis repetições a cada dose avaliada.

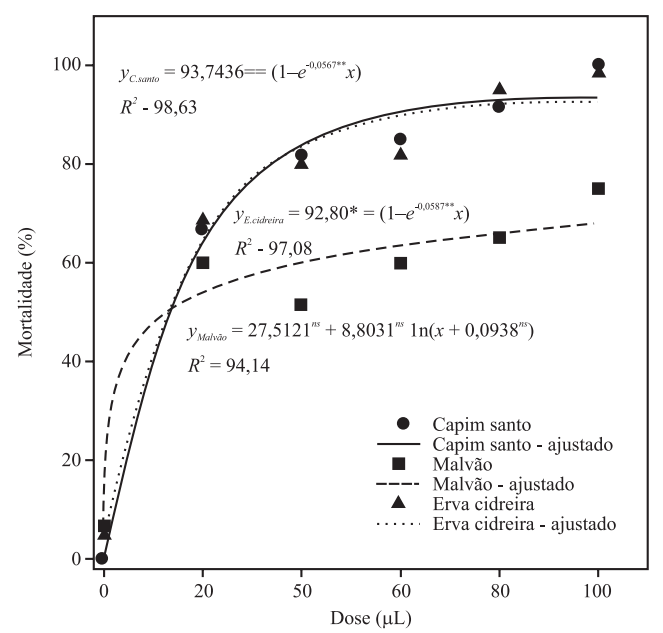

Figura 2 - Mortalidade de adultos de Callosobruchus maculatus (F.) submetidos a exposição aos óleos essenciais de Capim-santo (Cymbopogon citratus), Erva cidreira brasileira (Lippia alba) e extrato etanólico de Malvão (Plectranthus amboinicus) nas doses de 0, 20, 40, 60, 80 e $100 \mu \mathrm{L}$ ao longo de $24 \mathrm{~h}$ de exposição. Os símbolos representam as médias de seis repetições a cada dose avaliada. 
Tabela 1 - Mortalidade de adultos de Callosobruchus maculatus (F.) submetidos a exposição aos óleos essenciais de Capim-santo (Cymbopogon citratus), Erva cidreira brasileira (Lippia alba) e extrato etanólico de Malvão (Plectranthus amboinicus) após 24 h de exposição.

\begin{tabular}{lc}
\hline Tratamentos $^{* *}$ & Mortalidade (\%) \\
\hline Capim-santo (Cymbopogon citratus) & $70,83 \mathrm{a}$ \\
Erva cidreira brasileira (Lippia alba) & $71,38 \mathrm{a}$ \\
Malvão (Plectranthus amboinicus) & $53,05 \mathrm{~b}$ \\
\hline DMS & 5,84 \\
CV (\%) & 15,96 \\
\hline
\end{tabular}

${ }^{* *}$ ou ${ }^{*} P \leq 0,01$ ou 0,05 : significativo a $1 \%$ ou $5 \%$ de probabilidade, respectivamente, pelo teste- $F$. ${ }^{\text {ss: }}$ não significativo; DMS: diferença mínima significativa.

Médias na coluna seguidas de mesma letra minúscula são estatisticamente iguais de acordo com o teste de Tukey a $5 \%$ de probabilidade.

Ultimamente o interesse pelos produtos botânicos para o controle de pragas tem aumentado. Inúmeros estudos evidenciam danos à saúde humana decorrentes do uso de métodos de controle químico de insetos-praga. Entre eles, encontram-se doença de Alzheimer (Zaganas et al., 2013), com danos ao sistema imunológico (Corsini et al., 2013), ao sistema endócrino (Mostafalou \& Abdollahi, 2013), problemas neuropsicológicos e déficits neurológicos (Bjöjorling-Poulsen et al., 2008). Além destes problemas, ainda existe o risco de desenvolvimento de resistência das pragas aos inseticidas quando utilizados de forma contínua sem obedecer a doses adequadas (Pimentel et al., 2009; Song et al., 2011).

Levando em consideração a necessidade de minimizar os danos ao homem e ao meio ambiente causados pela intoxicação por resíduos químicos, bem como os grandes danos ocasionados pelo $C$. maculatus em feijão caupi, nosso estudo apontou que todas as espécies avaliadas apresentaram efeito repelente e potencial inseticida, causando mortalidades dos insetos acima de 50\% e Índices de repelência de aproximadamente $100 \%$.

Para a espécie $C$. citratus foi constatado elevado índice de repelência em todas as doses utilizadas, sendo que quando aplicado nas doses de 60 e $80 \mu \mathrm{L}$ mostrou-se mais eficiente. $\mathrm{O}$ menor IR dessa espécie foi observado na dose de $100 \mu \mathrm{L}$, porém, vale ressaltar que na menor dose utilizada do óleo essencial de Capim-santo já repeliu os insetos, evidenciando sua capacidade repelente. O Capimsanto também mostrou-se eficiente causando mortalidade superior a 70\% dos insetos no período de 24 h. Nossos resultados diferem dos encontrados por Araújo (2010), que constataram efeito repelente de $51 \%$ e apenas $20 \%$ de mortalidade de adultos de Zabrotes subfasciatus em grãos de feijão tratados com pó vegetal de C. citratus.

É possível afirmar que os resultados aqui apresentados para esta espécie se deva ao citral, uma vez que este componente do óleo essencial é composto pela mistura dos isômeros geranial e neral (Leal et al., 2003; Santos et al., 2009), além de limoneno, citronelal, mirceno e geraniol (Guerra et al., 2000; Santos et al., 2009), que podem ser os responsáveis pela ação do óleo essencial desta espécie no controle dos insetos. Além do mais, o óleo essencial traz maior concentração dos princípios ativos, quando comparado apenas ao uso do extrato seco das folhas, o que confere maior eficácia no controle aos artrópodes.

A Erva Cidreira Brasileira apresentou índice de repelência de aproximadamente $100 \%$ em todas as doses analisadas, mostrando-se superior as demais espécies nas doses acima de $40 \mu \mathrm{L}$ (Figura 1). O óleo essencial da $M$. officinalis possui importantes propriedades, sendo que a principal característica terapêutica da planta é sua adoção como calmante, atuando no sistema nervoso central através da modulação de processos cognitivos e do humor, além de alterar movimentos espontâneos (Kennedy et al., 2002; Akhondzadeh et al., 2003). Assim a Erva Cidreira Brasileira é amplamente utilizada como planta medicinal, característica esta que pode estar relacionada ao efeito repelente e de mortalidade do óleo essencial sobre os insetos estudados.

O efeito repelente da Erva Cidreira Brasileira, possivelmente, se deve ao citral presente em maior quantidade no óleo essencial extraído desta espécie. O citral pertence a principal classe de compostos que formam o óleo essencial da M. officinalis, os terpenos (Hay \& Waterman, 1993; Colussi et al., 2011). Além do deste, outros componentes presentes na folha são citronelal, $\beta$-cariofileno, germancreno, ocimeno e citronelol (Simões et al., 1998; Lorenzi \& Matos, 2002). Assim, é possível, ainda, atribuir a ação conjunta do citral com os outros componentes presentes nas folhas a toxicidade apresentada por esta espécie aos insetos estudados, ocasionando elevada mortalidade.

O extrato etanólico de Malvão (P. amboinicus) foi eficiente no que se refere à repelência dos insetos, principalmente na dose de $100 \mu \mathrm{L}$, apresentando IR de aproximadamente 70\% (Figura 1). Quanto a mortalidade dos insetos, o malvão mostrou-se tóxico, assegurando mortalidade de 50\% da população de C. maculatus (Tabela 1). 
Bandeira et al. (2011) observaram em seus estudos elevada concentração de trans-cariofileno nos óleos essenciais de $P$. neochilus e $P$. amboinicus. O cariofileno é um sesquiterpeno sintetizado pelas plantas na rota metabólica dos terpenos e, segundo Haslam (1996), o óleo essencial rico neste composto pode ser empregado no tratamento de diversas doenças. Portanto, a ocorrência de cariofileno no óleo essencial pode estar relacionada ao uso tradicional de espécies vegetais do gênero Plectranthus contra as dores estomacais (Bocardi, 2008; Bandeira et al., 2011). Considerando as propriedades medicinais do óleo essencial das espécies do gênero ao qual o Malvão (P. amboinicus) pertence, pode-se atribuir o desempenho desta espécie em nosso estudo ao cariofileno, composto responsável pelo uso terapêutico das espécies do gênero Plectranthus.

Na Figura 2, podemos observar a resposta de mortalidade as doses dos óleos essenciais e ao extrato etanólico das espécies estudadas. Temos que os óleos essenciais de C. citratus e Lippia alba não diferiram entre si e ocasionaram mortalidades superiores a $90 \%$ na dose de $80 \mu \mathrm{L}$ e que o extrato etanólico de $P$. amboinicus foi inferior as demais espécies e mesmo administrando-se as maiores doses, no entanto, a mortalidade não foi superior a $60 \%$.

Para as doses letais dos óleos essenciais de C. citratus e Lippia alba, houve semelhança quanto as doses, sendo que a $\mathrm{DL}_{50}$ foi alcançada nas doses de 13,67 e 13,28 $\mu \mathrm{L}$, respectivamente (Tabela 2). Já para o extrato etanólico do Malvão, verificou-se a menor dose letal para $30 \%$ da população dos insetos $\left(\mathrm{DL}_{30}=1,2 \mu \mathrm{L}\right)$, quando comparada as demais espécies, obtendo-se uma $\mathrm{DL}_{50}$ $=12,89 \mu \mathrm{L}$ muito semelhante aquelas para C. citratus $\mathrm{e}$ Lipia alba; no entanto, para a mortalidade de 70 e $90 \%$ dos insetos, estimaram-se doses letais de $125 \mu \mathrm{L}$ e $1220 \mu \mathrm{L}$, respectivamente, evidenciando a necessidade de doses mais elevadas do extrato etanólico para ocasionar a morte de 70 e $90 \%$ da população de $C$. maculatus, quando comparado ao resultado obtido com a exposição dos insetos aos óleos essenciais de Capim-santo e Erva Cidreira (Tabela 2).

Em estudos desenvolvidos sobre a toxicidade de óleos essenciais de Eucalyptus spp. sobre C. maculatus, Brito et al. (2006) constataram que para ocasionar a mortalidade de $10 \%$ e $50 \%$ da população de C. maculatus, as doses letais do óleo essencial de E. staigeriana são inferiores a dose mais baixa testada $(5 \mu \mathrm{L})$, e que as doses de 25 até $8 \mu \mathrm{L}$ do óleo pode matar $90 \%$ dos carunchos num período de tempo de até 48 horas. Observaram, ainda, que para as espécies E. citriodora e E. globulus a partir de 16 e 22 horas, respectivamente, até as 48 horas finais houve $100 \%$ de mortalidade dos insetos em todas as doses. Estes resultados corroboram com nossos estudos, visto que as doses letais aumentaram à medida que se aumentaram as doses aplicadas.

Tabela 2 - Doses letais em $\mu \mathrm{L}$ de óleos essenciais de Capimsanto (Cymbopogon citratus), Erva cidreira brasileira (Lippia alba) e extrato etanólico de Malvão (Plectranthus amboinicus) que provocam mortalidades de 30, 50, 70 e $90 \%$ de Callosobruchus maculatus (F.).

\begin{tabular}{lcccc}
\hline \multirow{2}{*}{ Espécie } & $\mathrm{DL}_{30}$ & $\mathrm{DL}_{50}$ & $\mathrm{DL}_{70}$ & $\mathrm{DL}_{90}$ \\
\cline { 2 - 5 }$(\mu \mathrm{L})$ & \multicolumn{4}{c}{} \\
\hline $\begin{array}{l}\text { Capim-santo (Cymbopo- } \\
\text { gon citratus) }\end{array}$ & 7,0 & 13,67 & 24,21 & 57,0 \\
$\begin{array}{l}\text { Erva cidreira brasileira } \\
\text { (Lippia alba) }\end{array}$ & 6,7 & 13,28 & 24,21 & 59,76 \\
$\begin{array}{l}\text { Malvão (Plectranthus } \\
\text { amboinicus) }\end{array}$ & 1,2 & 12,89 & 125 & 1220 \\
\hline
\end{tabular}

Dessa forma, podemos observar que as espécies C. citratus, L. alba e P. amboinicus apresentam efeitos tanto de repelência quanto de mortalidade sobre $C$. maculatus, fato positivo do ponto de vista do controle da praga, pois em algumas espécies é constatado apenas efeito repelente ou de mortalidade, e em alguns casos ambos. $\mathrm{O}$ fato da espécie ser repelente faz com que o inseto não consiga buscar alimento e nem consiga realizar a postura de ovos junto aos grãos, de modo a reduzir os níveis futuros de infestação populacional. Assim, podemos inferir que os óleos essenciais de C. citratus, L. alba e o extrato etanólico de $P$. amboinicus apresentaram-se promissores para o controle, por repelência, de C. maculatus.

\section{CONCLUSÕES}

As espécies avaliadas neste estudo apresentaram efeito repelente e potencial inseticida, causando mortalidades acima de 50\% e Índices de Repelência (IR) de 60 a 100\% sobre o Callosobruchus maculatus (F.).

O óleo essencial de Lippia alba foi mais eficiente nas doses superiores a $40 \mu \mathrm{L}$ ocasionando maior IR.

Os óleos essenciais de C. citratus e Lippia alba ocasionaram mortalidades superiores a 70\%.

Observou-se que os óleos essenciais apresentaram toxidade aguda tópica para os gorgulhos Callosobruchus 
maculatus: Cymbopogon citratus ( $\left.\mathrm{DL}_{50}=13,67 \mu \mathrm{L}\right)$, Lippia alba $\left(\mathrm{DL}_{50}=13,28 \mu \mathrm{L}\right)$ e Plectranthus amboinicus $\left(\mathrm{DL}_{50}=\right.$ $12,89 \mu \mathrm{L})$.

Os óleos essenciais e o extrato etanólico das plantas estudadas apresentaram toxicidade ao gorgulho-dofeijão, podendo, dessa forma, serem utilizados no controle desta praga durante o armazenamento

\section{AGRADECIMENTOS}

Ao CNPq pelo consentimento das bolsas de iniciação científica.

\section{LITERATURA CITADA}

ARAÚJO, A. M. N. Bioatividade de espécies vegetais em relação Zabrotes subfasciatus (Boheman, 1833) (Coleoptera: Chrysomelidae: Bruchinae) em feijão (Phaseolus vulgaris L., 1753). Dissertação (Mestrado em Agronomia) - Universidade Federal de Alagoas, 2010.

AKHONDZADEH, S.; NOROOZIAN, M.; MOHAMMADI, M.; OHADINIA, S.; JAMSHIDI, A. H.; KHANI, M. Salvia officinalis extract in the treatment of patients with mild to moderate Alzheimer's disease: a double blind, randomized and placebo-controlled trial. Journal of Clinical Pharmacy and Therapeutics, v. 28, p. 53-59, 2003.

BANDEIRA, J. M.; BARBOSA, F. F.; BARBOSA, L. M. P.; RODRIGUES, I. C. S.; BACARIN, M. A.; PETERS, J. A.; BRAGA, E. J. B. Composição do óleo essencial de quatro espécies do gênero Plectranthus. Revista Brasileira de Plantas Medicinais, Botucatu, v. 13, n. 2, p. 157-164, 2011.

BARROSO, M. S. T. Óleo essencial de Schinus molle L.: extração por arraste a vapor, modelagem matemática e análise química. Rio Grande do Sul. In: 10 SALÃO DE INICIAÇÃO CIENTÍFICA PUCRS, 2009, Rio Grande do Sul. Disponível em: <http://www.pucrs.br/edipucrs/ XSalaoIC/Engenharias/Engenharia_Quimica/70631 MAUREAN_SALLY_TAVARES_BARROSO.pdf>. Acesso em 10 de setembro de 2018.

BJØRLING-POULSEN, M.; ANDERSEN, H.; GRANDJEAN, P. Potential developmental neurotoxicity of pesticides used in Europe. Environmental Health, v. 7, p. 50, 2008.

BOCARDI, J. M. B. Etnofarmacologia das plantas medicinais de céu azul e composição química do óleo essencial de Plectranthus neochilus Schltr. Dissertação
(Mestrado em Química Aplicada) - Universidade Estadual de Ponta Grossa, Ponta Grossa. 2008. 101p.

BRITO, J. P.; OLIVEIRA, J. E. M.; BORTOLI, S. A. Toxicidade de óleos essenciais de Eucalyptus spp. sobre Callosobruchus maculatus (Fabr., 1775) (Coleoptera: Bruchidae). Revista de Biologia e Ciências da Terra, v. 6, n.1, 2006.

COLUSSI, T. C.; DALMOLIN, L. F.; PACHTMANN, M.; FREITAS, G. B. L. Melissa officinalis L.: Características gerais e biossíntese dos principais metabólitos secundários. Revista de Biologia e Farmácia, v. 5, n. 2, p. 92-95, 2011.

CONTE, C. O.; LAURA, V. A.; BATTISTELLI, J. Z.; CESCONETTO, A. O.; SOLON, S; FAVERO, S. Rendimento de óleo essencial de alfavaca por arraste à vapor em Clevenger, em diferentes formas de processamento das folhas. Horticultura Brasileira, v. 19. (suplemento) 2001. CD-ROM.

CONTE, C. O.; FAVERO, S. Toxidade e repelência de óleos essenciais de menta e capim limão para o gorgulho do milho. Horticultura Brasileira, v. 19. (suplemento). 2001. CD-ROM.

CORRÊA, J. C. R.; SALGADO, H. R. N. Atividade inseticida das plantas e aplicações: revisão. Revista Brasileira de Plantas Medicinais, Botucatu, v. 13, n. 4, p. 500-506, 2011.

CORSINI, E.; SOKOOTI, M.; GALLI, C. L.; MORETTO, A.; COLOSIO, C. Pesticide induced immunotoxicity in humans: A comprehensive review of the existing evidence. Toxicology, v. 307, p. 123-135, 2013.

ESTRELA, J. L. V.; FAZOLIN, M.; CATANO, V.; ALÉCIO, M. R.; LIMA, M. S. Toxicidade de óleos essenciais de Piper aduncun e Piper hispidinervum em Sitophilus zeamais. Pesquisa Agropecuária Brasileira, v. 41, n. 2, p.217-222, 2006.

FERNANDES, E. T.; FAVERO, S. Óleo essencial de Schinus molle L. para o controle de Sitophilus zeamais Most.1855 (Coleoptera:Curculionidae) em milho. Revista Brasileira de Agroecologia, v. 9, n. 1 p. 225-231, 2014.

GUERRA, M. J. M.; BADELL, J. B.; ALBAJES, A. R. R; PÉREZ, H. B.; VALENCIA, R. M.; AZCUY, A. L. Evaluación toxicológica aguda de los extractos fluidos al 30 y 80\% de Cymbopogon citratus (D.C.) Stapf (Caña Santa). Revista Cubana de Plantas Medicinais, v. 5, p. 97101, 2000.

HASLAM, E. Natural polyphenols (vegetable tannins) as drugs and medicines: possible modes of action. Journal of Natural Products, v. 59, p. 205-15, 1996. 
HAY, R. K. M.; WATERMAN, P. G. Physiology. Volatile oil crops: their biology, biochemistry and production. Essex, England: Longman, Scientific \& Technical, p.23-46, 1993.

KENNEDY, D. O.; SCHOLEY, A. B.; TILDESLEY, N. T. J.; PERRY, E. K.; WESNES, K. A. Modulation of mood and cognitive performance following acute administration of Melissa officinalis (lemon balm). Pharmacology Biochemistry and Behavior, v. 72, p. 953-964, 2002.

KIM, S. I.; ROH, J. Y.; KIM, D. H.; LEE, H. S.; AHN, Y. J. Insecticidal activities of aromatic plant extracts and essential oils against Sitophilus oryzae and Callosobruchus chinensis. Journal of Stored Products Research, v. 39, p. 293-303, 2003.

LEAL, T. C. A. B.; FREITAS, S. P.; SILVA, J. F.; CARVALHO, A. J. C. Produção de biomassa e óleo essencial em plantas de capim cidreira [Cymbopogon citratus (DC.) Stapf] em diferentes idades. Revista Brasileira de Plantas Medicinais, v. 5, p. 61-64, 2003.

LIMA, M. P. L.; OLIVEIRA, J. V.; BARROS, R.; TORRES, J. B. Identificação de genótipos de caupi Vigna unguiculata (L.) Walp. resistentes Callosobruchus maculatus (Fabr.) a (Coleoptera: Bruchidae). Neotropical Entomology, n. 30, v. 2, p. 289- 295, jun. 2001.

LORENZI, H.; MATOS, F. J. A. Plantas medicinais no Brasil: nativas e exóticas. Nova Odessa, SP: Instituto Plantarum, p. 512, 2002.

MELO, B. A.; OLIVEIRA S. R.; LEITE, D. T.; BARRETO, C. F.; HELTON, S. S. Inseticidas botânicos no controle de pragas de produtos armazenados. Revista Verde de Agroecologia e Desenvolvimento Sustentável, v. 6, n. 4, p. 01-10, outubro/dezembro de 2011.

MENEZES, E. L. A. Inseticidas botânicos: seus princípios ativos, modo de ação e uso agrícola. Embrapa Agrobiologia, p. 58, Rio de Janeiro: 2005.

MOSTAFAlOU, S.; ABDOLLAHI, M. Pesticides and human chronic diseases: Evidences, mechanisms, and perspectives. Toxicology and Applied Pharmacology, v. 268, p. 157-177, 2013.

PIMENTEL, M. A.; FARONI, L. R. A.; GUEDES, R. N.; SOUSA, A. H.; TÓTOLA, M. R. Phosphine resistance in
Brazilian populations of Sitophilus zeamais Motschusky (Coleoptera: Curculionidae). Journal of Stored Products Research, v. 45, n.1, p. 71-74, 2009.

QUIRINO, J. R.; LACERDA FILHO, A. F. DE; DEMITO, A. Utilização do resfriamento artificial na armazenagem de grãos. Sistemas Operacionais de Pós Colheita. Disponível em: < http://www.sop.eng.br/pdfs/ c031d281fe918c6cb391aae5d81c674b.pdf>. Acesso em 15 ago de 2011.

SAITO, M. L.; SCRAMIN, S. Plantas aromáticas e seu uso na agricultura. EMBRAPA Meio Ambiente. p. 48. Jaguariúna, 2000.

SANTOS, A.; PADUAN, R.H.; GAZIN, Z. C.; JACOMASSI, E.; D’ OLIVEIRA, P. S.; CORTEZ, D. A. G.; CORTEZ, L. E. R. Determinação do rendimento e atividade antimicrobiana do óleo essencial de Cymbopogon citratus (DC.) Stapf em função de sazonalidade e consorciamento. Revista Brasileira de Farmacognosia, p. 436-441, 2009.

SCHEEPENS, P.; HOEVERS, R.; ARULAPPAN, F. X.; PESCH, G. Armazenamento de produtos agrícolas. Wageningen. p. 85, 2011.

SILVA, S. Z. Resistência e qualidade tecnológicas de cultivares de feijão-caupi (Vigna unguiculata (L.) Walp) A Callosobruchus maculatus (F.) (Coleoptera: Bruchidae). Dissertação (Mestrado em Engenharia Agrícola; Sistemas Agroindustriais) Universidade Estadual do Oeste do Paraná. Cascavel, 2011.

SIMÕES, C. M. O.; MENTZ, L. A.; SCHENKEL, E. P.; IRGANG, B. E.; STEHMANN, J. R. Plantas da medicina popular no Rio Grande do Sul. 5. ed., Porto Alegre: UFRGS, 1998.

SONG, X.; WANG, P.; ZHANG, H.; Phosphine resistance in Rhyzopertha dominica (Fabricius) (Coleoptera: Bostrichidae) from different geographical populations in China. African Journal of Biotechnology, v. 10 p. 1636716373, 2011.

ZAGANAS, I; KAPETANAKI, S.; MASTORODEMOS, V.; KANAVOURAS, K., COLOSIO, C.; WILKS, M. F.; TSATSAKIS, A. M. Linking pesticide exposure and dementia: What is the evidence? Toxicology, v. 307, p. 3-11, 2013.

Recebido para publicação em 21/11/2018 e aprovado em 26/06/2019. 